\title{
Thrombocytopenia Should not be Barrier for Lymph-node Biopsy
}

\author{
Lili WU, Lanping DIAO, Lihong LIU, Guangyu MA, Chen HUANG, Yingzhen YAO, \\ Xiaolin WU, Guimin ZHAO
}

Hebei Medical University, Department of Hematology, the Fourth Hospital of Shijiazhuang, CHINA

To the Editor,

Enlargement of the lymph nodes occurs in various diseases. Pathological examination by lymph-node biopsy (LNB) is the primary method for the clinical diagnosis. In practical terms, however, many patients with enlarged lymph nodes also have severe thrombocytopenia. The risk of postoperative bleeding is very real, thereby making LNB hazardous and affecting the diagnostic workup.

Three patients with enlarged lymph nodes and severely reduced numbers of platelets were referred to the Department of Hematology of the Fourth Affiliated Hospital of Hebei Medical University. LNB was carried out for all patients, and patients received the definitive diagnosis and further treatment, as described here.

\section{CASE 1}

A 72-year-old male presented to the Department of Hematology of the Fourth Affiliated Hospital of Hebei Medical University with a 1- month history of intermittent fever and 7-day history of enlargement of multiple lymph nodes. He had been undergoing anti-infective therapy but without improvement. His medical history was unremarkable. Physical examination revealed multiple, painless enlarged lymph nodes in the neck, armpit and groin measuring about $0.5 \mathrm{~cm}$ to $1 \mathrm{~cm}$ in diameter but no other abnormal findings. Computed tomography of the cervical neck, thorax, abdomen, and pelvis demonstrated multiple small lymph nodes (diameter, $<10 \mathrm{~mm}$ ) in bilateral supraclavicular, axillae, mediastinum, retroperitoneal and inguinal regions. Simultaneously, multiple intumescent lymph nodes were observed alongside bilateral iliac blood vessels. Routine analyses of blood samples showed normal counts for leukocytes and platelets but decreased levels of hemoglobin $(\mathrm{Hb} ; 10.6 \mathrm{~g} / \mathrm{dl})$. The primary diagnosis was malignant lymphoma. In-depth consultations with the surgical team focused on LNBs, but they felt that repeat high fevers and chills were relative contraindications to surgery. They proposed to continue anti-infective treatment until the fever abated. Treatment with broad-spectrum antibiotics for a high fever was not effective and his condition worsened. Platelet count (PC) declined gradually to $1 \times 10^{9} / 1$ but hemorrhage was not detected. We suspected that the high fever and declining platelet count was linked to lymphoma. We negotiated with the surgical team repeatedly and communicated fully with the patient's family.

Eventually, LNBs on the right side of inguinal region were carried out after platelet transfusion. Complications (hematoma, bleeding at incision site) were not observed and the incision site healed well.

The histological diagnosis was angioimmunoblastic T-cell lymphoma (AITL). After one course of chemotherapy (CHOP regimen), the clinical features improved markedly with defervescence, shrinking of enlarged lymph nodes, and increase in platelet count to normal levels. 
Hereafter, he received multiple courses of chemotherapy but died of disease progression 2 years later.

\section{CASE 2}

A 70-year-old male was admitted to our hospital with a 4-month history of multiple enlarged lymph nodes with panhematopenia. Routine examination of blood samples repeatedly showed leukopenia (white blood cell (WBC) count, $2.4 \times 10^{9} / \mathrm{L}$ ), anemia(Hb: $8.8 \mathrm{~g} / \mathrm{dl})$ and with low $\mathrm{PC}\left(36 \times 10^{9} / \mathrm{L}\right)$. Bone-marrow biopsy led us to suspect T-cell lymphoma. He suffered irregular high fever and the platelet count fell to $<10 \times 10^{9} / \mathrm{L}$. Because of the risk of bleeding, LNB was not carried out in another hospital. Physical examination revealed scattered hemorrhagic spots on both lower extremities. Generalized, firm, painless enlarged lymph nodes (diameter, 1-6 cm) under the right ear, submandibularregion, both sides of the neck and supraclavicular region, as well as the right side of axillary and bilateral inguinal regions were noted. No other abnormalities were noted. Routine examination of blood samples showed WBC of $0.69 \times 10^{9} / \mathrm{L}$, neutrophilic granulocytes of $0.10 \times 10^{9} / \mathrm{L}, \mathrm{Hb}$ of $6.02 \mathrm{~g} /$ $\mathrm{dl}$, and $\mathrm{PC}$ of $7 \times 10^{9} / \mathrm{L}$. Coagulative functions were normal. CT of the head, cervical, chest and abdomen suggested multiple lymphadenectasis located laterally on both sides of the vessels of the neck, collar, axillary fossa and mediastina; roots of mesentery blood vessels; retroperitoneum; along iliac blood vessels. CT also showed splenomegaly and manipulus in the pelvic cavity. A bone-marrow smear showed karyotype hypoplasia, and the percentage of atypical lymphocytes was increased (74\%). He had been treated with an intravenous regimen of antibiotics (carbapenems) for prolonged fever, along with multiple transfusions of blood components for anemia and severe thrombocytopenia, without improvement. With a suspected diagnosis of lymphoma, we suggested that his symptoms were associated with this disease. After consultation with his family, a right cervical LNB was carried out. Bleeding was observed at the incision site during and after the incision was made. The patient remained pyrogenic and mucocutaneous bleeding was aggravated. WBC count, $\mathrm{Hb}$ level, and PC were decreased severely. Platelet transfusions were ineffective. On postoperative day-5, blurred vision was accompanied by a loss of consciousness and the patient died of spontaneous intracerebral hemorrhage. Three days later, the Pathology Department sent the result of histological examination of a lymph-node specimen: a diagnosis of AITL was recorded.

\section{CASE 3}

A 54-year-old male presented with a 1-month history of intermittent fever, fatigue, sweating and weight loss. At a county-level hospital, blood analyses revealed anemia (Hb: $6.0 \mathrm{~g} / \mathrm{dl}$ ) and thrombocytopenia (PC $38 \times 10^{9} / \mathrm{L}$ ) with a normal cell count. He had received erythrocyte transfusions and initial empiric treatment with antibiotics. The fever had resolved completely, but anemia had not. Medical history revealed splenectomy 6 years previously that led to the anemia and thrombocytopenia with massive splenomegaly that reached the level of the umbilicus. Results of pathological examination of the removed spleen were not provided. Upon physical examination, a severe anemic appearance was noted. However, hemorrhage tendency at the skin or mucosal membranes was not found. The superficial lymph nodes could not be palpated. An obsolete operation scar on left upper quadrant of the abdomen was noted. Results of the remainder of the examination were unremarkable. Blood analyses revealed a normal WBC count but increased lymphocytes (59-73\%), anemia (Hb, 5.6$6.0 \mathrm{~g} / \mathrm{dl}$ ) and thrombocytopenia (PC,13-20×10\%/L). Coagulative functions were normal. Percentage of abnormal lymphocytes in a bone-marrow smear was $34 \%$. Cellular morphology of abnormal lymphocytes was similar to that of hairy cells. Bonemarrow biopsy revealed that lymphomatoid cells had infiltrated the bone marrow but myelofibrosis was absent. CT of the thorax and abdomen showed enlargement of multiple lymph nodes with partial fusion in the mediastina and retroperitoneum. Color ultrasonography of the neck revealed several enlarged lymph nodes (diameter, $0.7-1.5 \mathrm{~cm}$ ) located laterally to right cervical vascular and supraclavicular regions. Because we suspected malignant lymphoma, we discussed LNB with the patient's 
family. Transfusions of blood components were given and right cervical LNB undertaken. The incision was not accompanied by obvious hemorrhage. Histopathological and immunohistochemical examinations revealed a low-grade malignant small B-cell lymphoma. The phenotype needed to be ascertained but the patient and family members refused further examinations. Combined with medical history (anemia, thrombocytopenia, massive splenomegaly) and pathology data, a high possibility of low-grade malignant small B-cell lymphoma was diagnosed. The patient had had three courses of chemotherapy but symptoms have not improved.

\section{DISCUSSION}

The diagnosis of lymphoma is confirmed by clinical, histological and immunohistochemical findings. Tissue samples are harvested by incisional biopsy, fine-needle aspiration biopsy (FNAB) and core-needle biopsy (CNB) ${ }^{1,2}$ Because of the complexity of the pathology of lymphoma and lack of sufficient tissue, most pathologists think that FNAB and CNB alone are not suitable for evaluation of lymphoproliferative disorders open surgical biopsy cannot be undertaken. ${ }^{3,4}$ Hence, excisional biopsy of lymph nodes remains the main method for the accurate classification of lymphoproliferative disorders. However, if patients have thrombocytopenia, postoperative hemorrhage is likely. Some patients with suspected lymphoma usually have severe thrombocytopenia caused by immunemediated bone-marrow infiltration, concurrent infection, and other factors. ${ }^{5-7}$ Patients and their relatives worry about the risks of intraoperative/ postoperative bleeding and refuse incisional biopsy, making the diagnosis and treatment difficult.

LNBs are not usually carried out if thrombocytopenia is present. The three cases presented here had severe thrombocytopenia and platelet transfusion was not effective. Case 2 was not diagnosed definitely for 4 months at another hospital, thereby wasting valuable time for treatment. Eventually, the LNB was under taken in all three cases for the diagnosis after close collaboration among patients, relatives, physicians, and surgical teams. Abiding by consensus guidelines for patients with throm- bocytopenia is important ${ }^{8}$, but LNBs could be safe for some patients because their fear of a delayed diagnosis or misdiagnosis will be considerable. ${ }^{9}$

For such patients, four strategies are very important. The first strategy is to improve laboratory examinations (especially bone-marrow aspiration to eliminate primary hematological malignancies). Secondly, if thrombocytopenia cannot be corrected after conventional therapy and the primary disease cannot be identified, the patient could undergo invasive procedures as long as he/she does not have a spontaneous-bleeding tendency so that specific treatment can be directed. Thirdly, the pathogenetic condition should be discussed fully with the patient's family to obtain written informed consent to obtain the diagnosis and initiate treatment. Finally, effective communication with the attending physician and surgical team is crucial.

The attending physician is often faced with a dilemma in arriving at the correct diagnosis and choice of optimal treatment. The attending physician must follow consensus guidelines but try his/ her best for the patient. The case studies shown here will help the attending physiciancaring for a patient with thrombocytopenia to make the correct decision for the diagnosis of the underlying condition.

\section{REFERENCES}

1. Alves MG, Chagas LR, Carvalho YR, et al. Metastatic melanoma of the tongue: a case report with immunohistochemical profile. Gerodontology 31: 314-319, 2014.

2. Frederiksen JK, Sharma M, Casulo C, Burack WR. Systematic review of the effectiveness of fine-needle aspiration and/or core needle biopsy for subclassifying lymphoma. Arch Pathol Lab Med 139: 245-251, 2015.

3. Amador-Ortiz C, Chen L, Hassan A, et al. Combined core needle biopsy and fine-needle aspiration with ancillary studies correlate highly with traditional techniques in the diagnosis of nodal-based lymphoma. Am J Clin Pathol 135: 516- 524, 2011.

4. Zelenetz AD, Gordon LI, Wierda WG, et al. Non-Hodgkin's lymphomas, version 2.2014. J Natl Compr Canc Netw 12: 916-946, 2014

5. Gebhart J, Lechner K, Skrabs C, et al. Lupus anticoagulant and thrombosis in splenic marginal zone lymphoma. Thromb Res 134: 980-984, 2014. 
International Journal of Hematology and Oncology

6. Yang $S M$, Jiang $Q$, Jiang $B$, et al. Clinical characteristics of splenic marginal zone lymphoma with abnormal complete blood count. Zhongguo Shi Yan Xue Ye Xue Za Zhi 21: 87-94, 2013.

7. Senapati J, Devasia AJ, David S, et al. Diffuse large B cell lymphoma in wiskott-Aldrich syndrome: a case report and review of literature. Indian J Hematol Blood Transfus 30 (Suppl 1): 309-313, 2014.

8. Thrombosis and Hemostasis group, Hematology Society, Chinese Medical Association. Consensus of Chinese experts on diagnosis and treatment of thrombotic thrombocytopenic purpura (version 2012). Zhonghua Xue Ye Xue Za Zhi 33: 983984, 2012

9. Pengjiang L, Mingfeng Z, Qi D, et al. Retrospective analysis of 86 patients with cytopenia during pregnancy. Zhonghua Xue Ye Xue Za Zhi 35: 71-72, 2014.

\section{Correspondence:}

Dr. Guimin ZHAO

Hebei Medical University

Fourth Hospital

Department of Hematology

050011 Shijiazhuang, CHINA

Tel.: t86 31186096334

Fax: t86 31186077634

E-mail: lily770215@163.com 